\title{
Theory of shear-induced migration in dilute polymer solutions near solid boundaries
}

\author{
Hongbo Ma and Michael D. Graham ${ }^{\mathrm{a})}$ \\ Department of Chemical and Biological Engineering, University of Wisconsin-Madison, Madison, \\ Wisconsin 53706-1691
}

(Received 19 November 2004; accepted 12 July 2005; published online 18 August 2005)

\begin{abstract}
In this work, a continuum theory is developed for the behavior of flowing dilute polymer solutions near solid surfaces, using a bead-spring dumbbell model of the dissolved polymer chains. Hydrodynamic interactions between the chains and the wall lead to migration away from the wall in shear flow. At steady state, this hydrodynamic effect is balanced by molecular diffusion; an analytical expression for the resulting concentration profile is derived. It is shown that the depletion layer thickness is determined by the normal stresses that develop in flow and can be much larger than the size of the polymer molecule. The transient development of this depletion layer is also studied, as well as the spatial development downstream from an entrance. Numerical and similarity solutions in these cases show that the developing concentration profile generally displays a maximum at an intermediate distance from the wall. (C) 2005 American Institute of Physics.
\end{abstract} [DOI: $10.1063 / 1.2011367]$

\section{INTRODUCTION}

The dynamics and transport of polymer solutions near surfaces or confined to small geometries are long-standing research topics that continue to pose many outstanding questions. Aside from their importance for traditional applications such as lubrication, adhesion, polymer processing, and oil recovery (i.e., flow of polymer solutions through porous media), these questions have recently taken on renewed importance with the emergence of microfluidic approaches to chemical and biological analyses, particle synthesis, and reaction engineering. As a specific example, we note that emerging technologies for single-molecule analysis of DNA in micron and nanometer scale devices (e.g., Refs. 1-7) have fueled substantial interest in the structure and dynamics of confined solutions of DNA. ${ }^{8-15}$ Predictive methods capable of describing the conformation and motion of polymer chains in microfluidic geometries would be of considerable significance for the conception and design of such devices. Finally, new experimental tools such as the surface forces apparatus ${ }^{16}$ allow investigation of confinement effects down into the nanoscale region, and refinements in fabrication technology allow construction of "nanofluidic" devices with dimensions in the sub-100-nm regime.

Experiments directly or indirectly indicate that during shear flow, flexible polymer molecules in solution migrate away from solid boundaries, leading to the formation of depletion layers and apparent slip at the boundaries. ${ }^{17}$ These phenomena have obvious implications for adsorption and desorption of macromolecules at solid surfaces, as molecules that tend to migrate away from walls are unlikely to adsorb on them. Motivated by these considerations, the focus of the present work is the development of an analytical theory of

\footnotetext{
a) Author to whom correspondence should be addressed. Electronic mail: graham@engr.wisc.edu
}

dilute polymer solutions flowing near solid surfaces. With this theory we derive a closed-form expression for the steady-state depletion layer thickness. Furthermore, we describe both the transient development of this depletion layer in uniform plane shear flow and the spatial development of the depletion layer downstream of the entrance to a channel. The end result is a general framework for understanding and prediction of migration phenomena in dilute polymer solutions.

\section{BACKGROUND}

Molecular migration in flowing dilute polymeric solutions is a well-known phenomenon that has received a significant amount of experimental and theoretical investigations. Much of this is reviewed by Agarwal, Dutta, and Mashelkar, ${ }^{17}$ so we focus here on a few particularly relevant studies. A recent experimental study was performed by Horn, Vinogradova, Mackay, and Phan-Thien ${ }^{18}$ in which apparent slip in a "Boger fluid" (a dilute solution of a high molecular weight polymer in a highly viscous solvent) was inferred from measurements in a surface forces apparatus. The slip length $L_{s}$ (the distance beyond the solid surface at which the velocity extrapolates to zero) was estimated to be three to five times the radius of gyration of the polymer (here polyisobutene). In a simple model of depletion layers, where the layer consists of pure solvent with a sharp change to the bulk polymer concentration at a distance $L_{d}$ from the wall, the relationship between slip length $L_{s}$ and depletion layer thickness $L_{d}$ is simply

$$
L_{s}=L_{d}(1-\beta) / \beta \text {, }
$$

where $\beta$ is the ratio of solvent viscosity to solution viscosity. Therefore, for a dilute solution, where $1-\beta \ll 1$, the depletion layer thickness is expected to be significantly larger than the slip length. As an example of a more classical study, 
Cohen and Metzner ${ }^{19}$ performed careful capillary flow experiments with nondilute polymer solutions, finding depletion layer thicknesses (using the formula above) up to eight times the polymer radius of gyration (other studies have found even larger values ${ }^{17}$ ). Additionally, these authors observed a direct correlation between the depletion layer thickness and the degree of elasticity of the polymer solution. Finally, Fang, Hu, and Larson ${ }^{20}$ have recently reported direct observations, using fluorescence microscopy, of large DNA molecules in shear flow near a solid surface. Their results clearly indicate the presence of a depletion layer, whose thickness increases with increasing shear rate and can be more than ten times the radius of gyration of the molecule.

A number of researchers have performed computational and theoretical studies of flowing polymer solutions near boundaries. Studies prior to 1994 have been extensively reviewed by Agarwal, Dutta, and Mashelkar. ${ }^{17}$ In a nonhomogeneous flow, the deformation and alignment of the polymer molecules are position dependent. Garner and Nissan ${ }^{21}$ proposed that the corresponding spatial variation in free energy could drive cross-streamline migration. Later, Marrucci ${ }^{22}$ related the entropy change with the stress level for an Oldroyd-B liquid and Metzner ${ }^{23}$ employed this result to analyze polymer retention in flows through porous media. Tirrell and Malone ${ }^{24}$ have made similar arguments. However, Aubert, Prager, and Tirrell ${ }^{25}$ pointed out that it is not clear that a spatial gradient in intramolecular free energy can result in displacement of the center of mass. Indeed, no such effect is found in first-principles kinetic theory developments for dilute solutions. Phenomenological two-fluid models have also been widely used to study migration and concentration fluctuations in polymer solutions at finite concentration. ${ }^{26-32}$ In these models, a contribution to the polymer mass flux proportional to $\boldsymbol{\nabla} \cdot \boldsymbol{\tau}^{p}$ is found, where $\tau^{p}$ is the polymer contribution to the stress tensor. Turning to the molecular kinetic theory point of view for (infinitely) dilute solutions, Aubert and Tirrell ${ }^{33}$ modeled the polymer as a flexible dumbbell in a viscous solvent and pointed out an effect in a nonhomogeneous flow field where the macromolecules lag behind the solvent motion along the streamline. In some kinetic theory developments, a contribution to the polymer flux corresponding to the divergence of the stress is found, ${ }^{34-37}$ which is similar to the result from the two-fluid models. ${ }^{30}$ However, the above arguments only lead to migration in a nonhomogeneous flow field. Furthermore, Curtiss and Bird ${ }^{38}$ pointed out that in the dilute solution kinetic theory results containing the divergence of the stress, the sum of the mass fluxes over all species is not zero, violating mass conservation and thus indicating a flaw in those developments.

In another approach to explaining the existence of depletion layers near confining surfaces, a number of researchers have amended theories by incorporating boundary effects, specifically the fact that polymer segments cannot pass through a solid wall. A typical method is treating the wall effect on the polymer molecules as a short-range purely repulsive potential. ${ }^{39}$ A refined version of this wall exclusion effect is provided by Mavrantzas and Beris ${ }^{40-42}$ and Woo, Shaqfeh, and Khomami ${ }^{14,15}$ where the change of the polymer chain statistics due to the wall is explicitly considered. How- ever, including this effect, the depletion layer thickness is still only on the order of the polymer molecule size, and would be insensitive to the flow strength, in contrast with the experimental observations.

A significant limitation of all the above-mentioned dilute solution studies is the neglect of intramolecular hydrodynamic interactions and the effect of the walls on the hydrodynamics of the solvent. If hydrodynamic interactions (HI) between polymer segments are ignored entirely, then no migration is found in shear flows without streamline curvature (plane shear flows, capillary, flow, etc.). If HI are included, but not their modifications due to the presence of a wall, then migration toward regions of higher shear rate is found; this is opposite to the trend observed experimentally. ${ }^{12}$ For example, Sekhon, Armstrong, and Jhon ${ }^{43}$ considered bulk hydrodynamic interactions in rectilinear slit flow using kinetic theory for a bead-spring dumbbell model, and concluded that cross-stream migration is possible with $\mathrm{HI}$, and Brunn ${ }^{44,45}$ and Brunn and $\mathrm{Chi}^{46}$ predicted migration toward the walls using Oseen-Burgers free-space hydrodynamic interactions for a bead-spring chain model. To our knowledge, only two studies in the polymer literature aside from our own (discussed below) have addressed the effect of hydrodynamic interactions in wall-bounded flows of dilute polymer solutions. Jhon and Freed ${ }^{47}$ incorporated a highly approximate representation of the near-wall hydrodynamics into a kinetic theory analysis for bead-spring polymer chains containing further approximations, predicting (correctly) migration away from the wall in simple plane shear flow. This prediction, however, is the result of cancellation of errors-the approximation to the hydrodynamics that they used would actually lead to a prediction of migration toward the wall if the kinetic theory were done exactly. The other result that we are aware of is a direct simulation. Fan, Phan-Thien, Yong, Wu, and $\mathrm{Xu}^{48}$ used dissipative particle dynamics ${ }^{49-51}$ (DPD) to study the behavior of flexible polymers in rectilinear flow through microchannels and predicted very weak migration toward the walls-a minimum at the centerline of the concentration distribution, different from experimental observations. However, in contrast with the experiments, both the particle and channel Reynolds numbers in these simulations were much larger than 1 . As discussed below, the hydrodynamic interaction with the wall is the main driving force for migration, and if the Reynolds number is not small that effect will be absent-a polymer molecule moves a significant distance down the channel in the time it takes for hydrodynamic fluctuations to propagate to the channel walls, so hydrodynamically, the polymer does not see the wall, and thus does not migrate.

Although motion of suspended droplets is not the focus of the present work, it is relevant to note that their migration in flow has also received a fair amount of attention; the older literature in this area is reviewed by Leal. ${ }^{52}$ Starting with a rigid particle in a Newtonian fluid at zero Reynolds number, Chan and Leal ${ }^{53}$ perturbatively examined the effects of inertia, droplet deformability, and non-Newtonian fluid character. For a slightly deformable drop in a Newtonian fluid in zero Reynolds number uniform shear flow near a wall, the wall modification to the hydrodynamic interaction is the sole con- 
tribution to droplet migration. Chan and Leal, using far-field wall hydrodynamic interaction, found that drift was always away from the wall (in agreement with experiment). For pressure-driven flow, where the shear rate is nonuniform, they found that the first-order contribution to migration was due to the interaction with the gradient of the local shear rate, provided the shear rate changed significantly over the length scale of the droplet. They found that the direction of migration depended on the ratio of solvent and droplet viscosities; for the range of viscosity ratios used in experiments, migration was always toward regions of lower local shear rate. For circular Couette flow, they found that the final position of the droplet was determined from a competition between a streamline curvature effect and wall hydrodynamic interaction. An important observation was made by Smart and Leighton, ${ }^{54}$ who pointed out that a droplet far from a wall can be treated to leading order as a symmetric force dipole (stresslet) and that the wall-induced migration effect is due to the flow induced by the image of the dipole on the other side of the wall. This result generalizes to any particle or macromolecule in flow above a wall and plays an important role in the results described below.

The discussion of droplet dynamics makes clear the necessity to correctly account for hydrodynamic effects in studying the motion of flexible particles or macromolecules near solid boundaries. In prior work, we have developed a coarse-grained (bead-spring chain) model of long (>100 persistence length) double-stranded DNA, incorporating hydrodynamic interactions. The model provides an accurate representation of experimental data (structural and dynamic) for DNA in bulk solution, ${ }^{55,56}$ and has been extended to capture the dynamics of DNA solutions in microchannels, including hydrodynamic effects. ${ }^{10-12}$ Relaxation and diffusion of chains in a channel of square cross section ${ }^{10,11}$ follow the predictions of a simple scaling theory, due to Brochard and de Gennes, ${ }^{57}$ that is based on the screening of segmentsegment hydrodynamic interactions by the confining walls. Furthermore, the simulation results for diffusion in a slit channel (i.e., between parallel infinite walls) agree very well with experiments. ${ }^{58}$ More interestingly, the simulations predict that during pressure-driven flow in a channel, the molecules will tend to migrate toward the centerline, forming depletion layers that are much larger than the radius of gyration of the molecules. ${ }^{11,12,59}$ The goal of the present work is to complement those detailed simulations with theoretical results that provide a more fundamental understanding of the migration phenomenon.

\section{ILLUSTRATION OF MIGRATION MECHANISM}

To illustrate the basic mechanism of hydrodynamic migration of a dissolved polymer molecule in a confined geometry, we begin by considering a bead-spring dumbbell model of the polymer above a single wall. Hydrodynamically, each moving bead is treated as a point force acting on the fluid; ignoring for the moment the Brownian forces, the hydrodynamic forces introduced by the two beads must be equal and opposite, balancing the extension of the spring. The flow due to the motion of each bead (i.e., the solution to Stokes' equa-

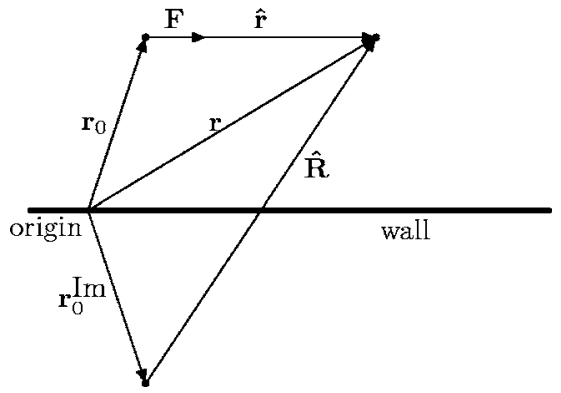

FIG. 1. Illustration of the position vectors used for a point force above a plane wall.

tion) is available in simple analytical form, ${ }^{60}$ thus allowing a complete description of the flow. In this section we will illustrate this flow; below we will build it into an analytical theory allowing prediction of the dynamics of depletion layer formation in flow.

Assuming the wall is at $y=0$, let $\mathbf{r}_{0}=\left(x_{0}, y_{0}, z_{0}\right)$ be the position of one bead and denote the distance vectors

$$
\begin{aligned}
& \hat{\mathbf{r}}=\mathbf{r}-\mathbf{r}_{0}, \\
& \hat{\mathbf{R}}=\mathbf{r}-\mathbf{r}_{0}^{\mathrm{Im}},
\end{aligned}
$$

where $\mathbf{r}_{0}^{\mathrm{Im}}=\left(x_{0},-y_{0}, z_{0}\right)$ is the mirror image of $\mathbf{r}_{0}$ with respect to the wall. The force exerted on the fluid due to the motion of this bead is $\mathbf{F}$. These vectors are shown in Fig. 1. The perturbation flow at any other position $\mathbf{r}(x, y, z)$ caused by the motion of the bead can be obtained by solving the Stokes' equation,

$$
0=-\nabla p+\eta \nabla^{2} \mathbf{v}+\delta\left(\mathbf{r}-\mathbf{r}_{0}\right) \mathbf{F},
$$

subject to no-slip boundary condition at the wall,

$$
\mathbf{v}\left(x, y=0, z ; \mathbf{r}_{0}\right)=0,
$$

where $\eta$ is the solvent viscosity, $\mathbf{v}$ is the velocity, and $p$ is the pressure. The solution has the following form:

$$
\begin{aligned}
& \mathbf{v}=\boldsymbol{\Omega} \cdot \mathbf{F}, \\
& \boldsymbol{\Omega}\left(\mathbf{r}, \mathbf{r}_{0}\right)=\frac{1}{8 \pi \eta}\left[\mathbf{S}(\hat{\mathbf{r}})-\mathbf{S}(\hat{\mathbf{R}})+2 y_{0}^{2} \mathbf{P}^{D}(\hat{\mathbf{R}})-2 y_{0} \mathbf{S}^{D}(\hat{\mathbf{R}})\right],
\end{aligned}
$$

where $\mathbf{S}$ is the free-space Stokeslet, $\mathbf{P}^{D}$ is the potential dipole, and $\mathbf{S}^{D}$ is the Stokeslet doublet. ${ }^{61}$ These are given, respectively, as

$$
\begin{aligned}
& S_{i j}(\mathbf{r})=\frac{\delta_{i j}}{r}+\frac{x_{i} x_{j}}{r^{3}}, \\
& P_{i j}^{D}(\mathbf{r})= \pm \frac{\partial}{\partial x_{j}}\left(\frac{x_{i}}{|\mathbf{r}|^{3}}\right)= \pm\left(\frac{\delta_{i j}}{|\mathbf{r}|^{3}}-3 \frac{x_{i} x_{j}}{|\mathbf{r}|^{5}}\right),
\end{aligned}
$$




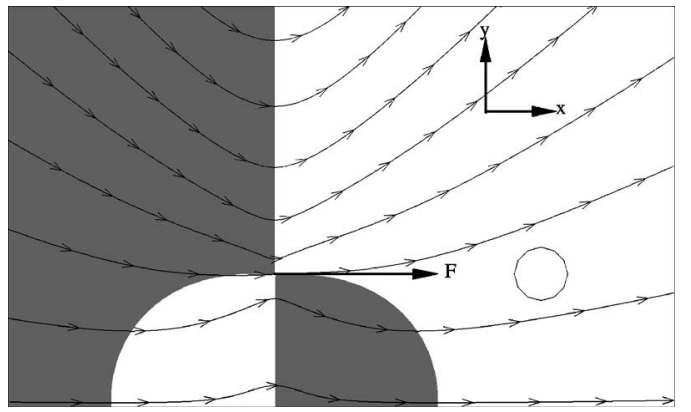

FIG. 2. Velocity field due to a point force in the $x$ direction located at $(x, y)=(-5 a, 5 a)$, where $a$ is the bead radius. The plane wall is at $y=0$. The lines correspond to streamlines, while the light and dark areas indicate regions where the wall-normal velocity is positive (away from the wall) and negative (towards the wall), respectively. Also shown is a "bead" of radius $a$ located at $(x, y)=(5 a, 5 a)$ - this can be thought of as the other end of a relaxing dumbbell oriented parallel to the wall.

$$
S_{i j}^{D}(\mathbf{r})= \pm \frac{\partial S_{i 2}}{\partial x_{j}}=x_{2} P_{i j}^{D}(\mathbf{r}) \pm \frac{\delta_{j 2} x_{i}-\delta_{i 2} x_{j}}{|\mathbf{r}|^{3}}
$$

with the minus sign for $j=2$ corresponding to the $y$ direction, and the plus sign for $j=1,3$ corresponding to the $x$ and $z$ directions. ${ }^{60}$

Using this solution, we calculate the velocity field caused by a point force parallel to the wall, which corresponds to one end of a relaxing dumbbell parallel to the wall. The flow field is shown in Fig. 2. It can be seen that the flow induced by one bead of the relaxing dumbbell will be upward at the position of the other bead, and vice versa. In other words, each bead will be convected away from the wall by the velocity perturbation caused by its partner. As a whole, the center-of-mass of the dumbbell migrates from the wall. In contrast, a relaxing dumbbell perpendicular to the wall will move toward the wall. A simple explanation of this result is that the mobility of the bead nearest the wall is lower than that of its partner. ${ }^{12}$ In shear flow, dumbbells are more likely to be oriented parallel to the wall. Thus, migration away from the wall is dominant.

\section{KINETIC THEORY FOR A DUMBBELL IN DILUTE SOLUTION}

The simple analysis in Sec. III predicts that a macromolecule near a wall will migrate due to hydrodynamic interaction with the wall, providing a starting point to explore many interesting phenomena. In this section, we will incorporate bead-wall hydrodynamic interactions in the polymer kinetic theory for a bead-spring dumbbell in solution to investigate the formation of the depletion layer in a flowing polymer solution near a solid wall.

Let $\mathbf{r}_{1}$ and $\mathbf{r}_{2}$ denote the position vectors of the two beads of a dumbbell. Then the position of the center-of-mass is $\mathbf{r}_{c}=\left(\mathbf{r}_{1}+\mathbf{r}_{2}\right) / 2$, and the connector vector is $\mathbf{q}=\mathbf{r}_{2}-\mathbf{r}_{1}$. The quantities $\dot{\mathbf{r}}_{c}$ and $\dot{\mathbf{q}}$ give the rate of change of the center-ofmass and the connector vector. The conservation equation for the probability distribution density function $\Psi\left(\mathbf{r}_{c}, \mathbf{q}, t\right)$ is ${ }^{62}$

$$
\frac{\partial \Psi}{\partial t}=-\frac{\partial}{\partial \mathbf{r}_{c}} \cdot\left(\dot{\mathbf{r}}_{c} \Psi\right)-\frac{\partial}{\partial \mathbf{q}} \cdot(\dot{\mathbf{q}} \Psi) .
$$

Integrating the above equation over $\mathbf{q}$ and defining

$$
\begin{aligned}
& \Psi\left(\mathbf{r}_{c}, \mathbf{q}, t\right)=n\left(\mathbf{r}_{c}, t\right) \hat{\Psi}\left(\mathbf{r}_{c}, \mathbf{q}, t\right), \\
& n\left(\mathbf{r}_{c}, t\right)=\int \Psi\left(\mathbf{r}_{c}, \mathbf{q}, t\right) d \mathbf{q},
\end{aligned}
$$

give the governing equation for the center-of-mass probability distribution ("concentration"), $n\left(\mathbf{r}_{c}, t\right)$,

$$
\frac{\partial n}{\partial t}=-\frac{\partial}{\partial \mathbf{r}_{c}} \cdot \mathbf{j}_{c},
$$

where $\mathbf{j}_{c}=\left\langle\dot{\mathbf{r}}_{c}\right\rangle n$ is the center-of-mass flux integrated over the internal degrees of freedom of the molecule, and the angle brackets designate an ensemble average over the configuration variable $\mathbf{q}$,

$$
\langle\mathbf{A}\rangle=\int A \hat{\Psi} d \mathbf{q} .
$$

The fluxes $\dot{\mathbf{r}}_{c} \Psi$ and $\dot{\mathbf{q}} \Psi$ in Eq. (11) are determined by a balance between the spring force $\mathbf{F}_{i}^{s}$, hydrodynamic force $\mathbf{F}_{i}^{h}$, wall repulsion force $\mathbf{F}_{i}^{w}$, and Brownian force $\mathbf{F}_{i}^{b}$ exerted on each bead,

$$
\mathbf{F}_{i}^{h}+\mathbf{F}_{i}^{s}+\mathbf{F}_{i}^{w}+\mathbf{F}_{i}^{b}=0, \quad i=1,2 .
$$

Assuming equilibrium in momentum space, the Brownian force is given by ${ }^{62}$

$$
\mathbf{F}_{i}^{b}=-k_{B} T \frac{\partial}{\partial \mathbf{r}_{i}} \ln \Psi,
$$

where $k_{B}$ is the Boltzmann constant and $T$ is the temperature. The hydrodynamic force $\mathbf{F}_{i}^{h}$ is proportional to the velocity difference between the bead $i$ and fluid, as given by Stokes' law. The actual form of the spring force here is arbitrary. In other words, the analysis given here applies to any spring law.

In our previous simulation work, ${ }^{12}$ which accounts for the wall exclusion force $\mathbf{F}_{i}^{w}$, we found that in flow, this effect is generally small relative to the hydrodynamic effect. In particular, although the static exclusion force acts over a range of about the polymer radius of gyration $R_{g}$, the hydrodynamic effect on the chains in flow leads to depletion over length scales much larger than $R_{g}$. Below, we further elucidate this phenomenon. This simulation result is consistent with many experiments ${ }^{20}$ that directly or indirectly indicate the existence of depletion layers with thicknesses much larger than the polymer molecule size, a result that cannot be accounted for by simple wall exclusion arguments. Therefore, in the following analysis we set $\mathbf{F}_{i}^{w}=0$.

Using Eq. (16), the velocity of the center of mass $\dot{\mathbf{r}}_{c}$ is given by

$$
\dot{\mathbf{r}}_{c}=\frac{1}{2}\left[\sum_{i=1}^{2} \mathbf{v}\left(\mathbf{r}_{i}\right)+\frac{1}{k_{B} T} \sum_{i=1}^{2} \sum_{j=1}^{2} \mathbf{D}_{i j} \cdot\left(\mathbf{F}_{j}^{s}+\mathbf{F}_{j}^{b}\right)\right] .
$$

In this equation, 


$$
\mathbf{D}_{i j}=k_{B} T\left(\frac{1}{6 \pi \eta a} \mathbf{I} \delta_{i j}+\mathbf{\Omega}_{i j}\right),
$$

where $a$ is the bead radius, $\mathbf{v}\left(\mathbf{r}_{i}\right)$ is the unperturbed flow velocity at the position of bead $i, \mathbf{I}$ is the unit tensor, $\delta_{i j}$ is the Kronecker delta, and $\boldsymbol{\Omega}_{i j}$ denotes the hydrodynamic interaction tensor,

$$
\boldsymbol{\Omega}_{i j}=\boldsymbol{\Omega}\left(\mathbf{r}_{i}, \mathbf{r}_{j}\right)-\frac{\delta_{i j}}{8 \pi \eta} \mathbf{S}\left(\mathbf{r}_{i}-\mathbf{r}_{j}\right),
$$

with $\boldsymbol{\Omega}\left(\mathbf{r}_{i}, \mathbf{r}_{j}\right)$ given in Eq. (7). The rate of change of the connector vector $\dot{\mathbf{q}}$ is given by

$$
\dot{\mathbf{q}}=\dot{\mathbf{r}}_{2}-\dot{\mathbf{r}}_{1}=\left[\mathbf{v}\left(\mathbf{r}_{2}\right)-\mathbf{v}\left(\mathbf{r}_{1}\right)\right]+\sum_{j=1}^{2}\left(\boldsymbol{\Omega}_{2 j}-\boldsymbol{\Omega}_{1 j}\right) \cdot\left(\mathbf{F}_{j}^{s}+\mathbf{F}_{j}^{b}\right) .
$$

Defining the spring force $\mathbf{F}^{s}=\mathbf{F}_{1}^{s}=-\mathbf{F}_{2}^{s}$ and using Eq. (17) along with

$$
\begin{gathered}
\frac{\partial}{\partial \mathbf{r}_{1}}=\frac{1}{2} \frac{\partial}{\partial \mathbf{r}_{c}}-\frac{\partial}{\partial \mathbf{q}}, \\
\frac{\partial}{\partial \mathbf{r}_{2}}=\frac{1}{2} \frac{\partial}{\partial \mathbf{r}_{c}}+\frac{\partial}{\partial \mathbf{q}},
\end{gathered}
$$

the velocity of the center-of-mass of the dumbbell can be expressed as

$$
\begin{aligned}
\dot{\mathbf{r}}_{c}= & \mathbf{v}+\frac{1}{8} \mathbf{q q}: \nabla \nabla \mathbf{v}+\frac{1}{2} \overline{\mathbf{\Omega}} \cdot \mathbf{F}^{s}+\frac{1}{2} \overline{\mathbf{D}} \cdot \frac{\partial}{\partial \mathbf{q}} \ln \Psi \\
& -\mathbf{D}_{k} \cdot \frac{\partial}{\partial \mathbf{r}_{c}} \ln \Psi,
\end{aligned}
$$

where

$$
\begin{aligned}
& \overline{\mathbf{\Omega}}=\left(\boldsymbol{\Omega}_{11}-\boldsymbol{\Omega}_{22}\right)+\left(\boldsymbol{\Omega}_{21}-\boldsymbol{\Omega}_{12}\right), \\
& \overline{\mathbf{D}}=k_{B} T \overline{\mathbf{\Omega}}, \\
& \mathbf{D}_{K}=\frac{1}{4}\left[\left(\mathbf{D}_{11}+\mathbf{D}_{22}\right)+\left(\mathbf{D}_{21}+\mathbf{D}_{12}\right)\right] .
\end{aligned}
$$

Here $\mathbf{v}$ is the unperturbed fluid velocity at the center of mass of the dumbbell, $\mathbf{r}_{c}$, and we have Taylor-expanded $\mathbf{v}\left(\mathbf{r}_{1}\right)$ and $\mathbf{v}\left(\mathbf{r}_{2}\right)$ around $\mathbf{r}_{c}$ and kept the terms up to the second order. This accounts for the difference, in a nonhomogeneous flow field, between the translational velocity of the center of mass of the dumbbell and the unperturbed fluid velocity at the position of the center of mass. The quantity $\mathbf{D}_{K}$ is the socalled Kirkwood diffusivity for a dumbbell. ${ }^{62}$ Multiplying Eq. (24) by $\Psi$, integrating over the internal coordinate $\mathbf{q}$, and using incompressibility, one can arrive at the mass flux expression,

$$
\begin{aligned}
\mathbf{j}_{c}= & n \mathbf{v}+\frac{n}{8}\langle\mathbf{q q}\rangle: \nabla \nabla \mathbf{v}+\frac{1}{2}\left\langle\overline{\boldsymbol{\Omega}} \cdot\left(\mathbf{F}^{\mathbf{s}}+k_{B} T \frac{\partial}{\partial \mathbf{q}} \ln \hat{\Psi}\right)\right\rangle n \\
& -\left\langle\mathbf{D}_{K} \cdot \frac{\partial \ln \hat{\Psi}}{\partial \mathbf{r}_{c}}\right\rangle_{n}-\left\langle\mathbf{D}_{K}\right\rangle \cdot \frac{\partial n}{\partial \mathbf{r}_{c}} .
\end{aligned}
$$

This expression is valid for an arbitrary flow geometry. A general discussion for the case of a dumbbell near a single wall is given by Jendrejack, Schwartz, de Pablo, and Graham. ${ }^{12}$ Here we simplify this expression by considering the case where the extension of the dumbbell $|\mathbf{q}|$ is small compared to its distance from the wall $y$; i.e., we focus on the far- field effects of the wall.

First, we define a reflection operator $\mathbf{T}$,

$$
\mathbf{T}=\delta-2 \mathbf{e}_{y} \mathbf{e}_{y}=\left(\begin{array}{ccc}
1 & 0 & 0 \\
0 & -1 & 0 \\
0 & 0 & 1
\end{array}\right)
$$

Then, the image positions of the two beads and the centerof-mass with respect to the wall are

$$
\begin{aligned}
& \mathbf{r}_{1}^{\mathrm{Im}}=\mathbf{T} \cdot \mathbf{r}_{1}, \\
& \mathbf{r}_{2}^{\mathrm{Im}}=\mathbf{T} \cdot \mathbf{r}_{2}, \\
& \mathbf{r}_{c}^{\mathrm{Im}}=\mathbf{T} \cdot \mathbf{r}_{c} .
\end{aligned}
$$

We also define a series of vectors:

$$
\hat{\mathbf{R}}_{c}=\mathbf{r}_{c}-\mathbf{r}_{c}^{\mathrm{Im}}=\mathbf{r}_{c}-\mathbf{T} \cdot \mathbf{r}_{c},
$$

$$
\begin{aligned}
& \hat{\mathbf{R}}_{11}=\mathbf{r}_{1}-\mathbf{r}_{1}^{\mathrm{Im}}=\hat{\mathbf{R}}_{c}-\frac{1}{2}(\mathbf{q}-\mathbf{T} \cdot \mathbf{q}), \\
& \hat{\mathbf{R}}_{22}=\mathbf{r}_{2}-\mathbf{r}_{2}^{\mathrm{Im}}=\hat{\mathbf{R}}_{c}+\frac{1}{2}(\mathbf{q}-\mathbf{T} \cdot \mathbf{q}), \\
& \hat{\mathbf{R}}_{12}=\mathbf{r}_{1}-\mathbf{r}_{2}^{\mathrm{Im}}=\hat{\mathbf{R}}_{c}-\frac{1}{2}(\mathbf{q}+\mathbf{T} \cdot \mathbf{q}), \\
& \hat{\mathbf{R}}_{21}=\mathbf{r}_{2}-\mathbf{r}_{1}^{\mathrm{Im}}=\hat{\mathbf{R}}_{c}+\frac{1}{2}(\mathbf{q}+\mathbf{T} \cdot \mathbf{q}), \\
& \hat{\mathbf{r}}_{\alpha \beta}=\mathbf{r}_{\alpha}-\mathbf{r}_{\beta} .
\end{aligned}
$$

Using this notation and Eq. (20), $\overline{\mathbf{\Omega}}$ can be rewritten as follows for flow above a single wall: 


$$
\begin{aligned}
\overline{\mathbf{\Omega}}= & \frac{1}{8 \pi \eta}\left\{\left[-\mathbf{S}\left(\hat{\mathbf{R}}_{11}\right)+2 y_{1}^{2} \mathbf{P}^{D}\left(\hat{\mathbf{R}}_{11}\right)-2 y_{1} \mathbf{S}^{D}\left(\hat{\mathbf{R}}_{11}\right)\right]-\left[-\mathbf{S}\left(\hat{\mathbf{R}}_{22}\right)+2 y_{2}^{2} \mathbf{P}^{D}\left(\hat{\mathbf{R}}_{22}\right)-2 y_{2} \mathbf{S}^{D}\left(\hat{\mathbf{R}}_{22}\right)\right]+\left[\mathbf{S}\left(\hat{\mathbf{r}}_{21}\right)-\mathbf{S}\left(\hat{\mathbf{R}}_{21}\right)\right.\right. \\
& \left.\left.+2 y_{1}^{2} \mathbf{P}^{D}\left(\hat{\mathbf{R}}_{21}\right)-2 y_{1} \mathbf{S}^{D}\left(\hat{\mathbf{R}}_{21}\right)\right]-\left[\mathbf{S}\left(\hat{\mathbf{r}}_{12}\right)-\mathbf{S}\left(\hat{\mathbf{R}}_{12}\right)+2 y_{2}^{2} \mathbf{P}^{D}\left(\hat{\mathbf{R}}_{12}\right)-2 y_{2} \mathbf{S}^{D}\left(\hat{\mathbf{R}}_{12}\right)\right]\right\} .
\end{aligned}
$$

For $|\mathbf{q}| \ll\left|\hat{\mathbf{R}}_{c}\right|$, we can Taylor expand $\overline{\mathbf{\Omega}}$ around $\hat{\mathbf{R}}_{c}$. Keeping only leading terms yields

$$
\begin{aligned}
\overline{\mathbf{\Omega}}= & \frac{1}{8 \pi \eta}\left\{-2[\mathbf{T} \cdot \mathbf{q}] \cdot \nabla \mathbf{S}+4 y^{2}[\mathbf{T} \cdot \mathbf{q}] \cdot \nabla \mathbf{P}^{D}\right. \\
& -4 y[\mathbf{T} \cdot \mathbf{q}] \cdot \nabla \mathbf{S}^{D}-8 y q_{y} \mathbf{P}^{D}\left(\hat{\mathbf{R}}_{c}\right)-4 q_{y} \mathbf{S}^{D}\left(\hat{\mathbf{R}}_{c}\right) \\
& +\cdots\} .
\end{aligned}
$$

The gradient terms are readily calculated from Eqs. (8)-(10), allowing Eq. (40) to be simplified further,

$$
\overline{\mathbf{\Omega}}=\frac{3}{32 \pi \eta} \frac{1}{y^{2}}\left(\begin{array}{ccc}
-q_{y} & -q_{x} & 0 \\
q_{x} & -2 q_{y} & q_{z} \\
0 & -q_{z} & -q_{y}
\end{array}\right)+\cdots .
$$

This can be rewritten compactly to leading order as

$$
\overline{\mathbf{\Omega}}=\frac{3}{32 \pi \eta y^{2}} \tilde{\mathcal{M}} \cdot \mathbf{q},
$$

where $\tilde{\mathcal{M}}$ is a third order tensor with the following components:

$$
\begin{aligned}
& \tilde{\mathcal{M}}_{222}=-2 \\
& \tilde{\mathcal{M}}_{211}=\tilde{\mathcal{M}}_{233}=1 \\
& \tilde{\mathcal{M}}_{121}=\tilde{\mathcal{M}}_{112}=\tilde{\mathcal{M}}_{323}=\tilde{\mathcal{M}}_{332}=-1, \\
& \tilde{\mathcal{M}}_{i j k}=0, \quad \text { i, } \mathrm{j}, \mathrm{k}=\text { others. }
\end{aligned}
$$

We will denote the tensor $\mathcal{M}=3 \tilde{\mathcal{M}} / 64 \pi \eta y^{2}$ as the migration tensor. Finally we point out here that this tensor can be defined for any geometry, given the point force solution for Stokes' equation in that geometry.

Similar to $\overline{\boldsymbol{\Omega}}$, the leading order $\mathbf{D}_{K}$ is given by

$$
\mathbf{D}_{K}=\frac{k_{B} T}{12 \pi \eta a}\left[\mathbf{I}+\frac{3 a}{4} \mathbf{S}(\mathbf{q})\right] .
$$

Recalling that the polymer contribution to the stress tensor $\tau_{p}$ (Ref. 62) is

$$
\tau^{p}=n\left\langle\mathbf{q} \mathbf{F}^{\mathbf{s}}\right\rangle-n k_{B} T \mathbf{I},
$$

and using Eqs. (42) and (47), we can simplify Eq. (28) at leading order to the following:

$$
\begin{aligned}
\mathbf{j}_{c}= & n \mathbf{v}+\frac{n}{8}\langle\mathbf{q q}\rangle: \nabla \nabla \mathbf{v}+\mathcal{M}: \tau^{p} \\
& -\frac{k_{B} T}{12 \pi \eta a}\left\langle\left(\mathbf{I}+\frac{3 a}{4} \mathbf{S}(\mathbf{q})\right) \cdot \frac{\partial \ln \hat{\Psi}}{\partial \mathbf{r}_{c}}\right\rangle_{n} \\
& -\frac{k_{B} T}{12 \pi \eta a}\left\langle\mathbf{I}+\frac{3 a}{4} \mathbf{S}(\mathbf{q})\right\rangle \cdot \frac{\partial n}{\partial \mathbf{r}_{c}} .
\end{aligned}
$$

Now we define

$$
\left\langle\mathbf{D}_{K, b}\right\rangle=\frac{k_{B} T}{12 \pi \eta a}\left\langle\mathbf{I}+\frac{3 a}{4} \mathbf{S}(\mathbf{q})\right\rangle .
$$

This is the bulk, ensemble-averaged (but conformation dependent) Kirkwood diffusivity. Finally, we use this to rewrite Eq. (49),

$$
\begin{aligned}
\mathbf{j}_{c}= & n \mathbf{v}+\frac{n}{8}\langle\mathbf{q q}\rangle: \nabla \nabla \mathbf{v}+\mathcal{M}: \tau^{p}-n \frac{\partial}{\partial \mathbf{r}_{c}} \cdot\left\langle\mathbf{D}_{K, b}\right\rangle \\
& -\left\langle\mathbf{D}_{K, b}\right\rangle \cdot \frac{\partial n}{\partial \mathbf{r}_{c}} .
\end{aligned}
$$

The last term in this expression is the normal Fickian diffusion; the other terms lead to migration. Consider first the term containing the migration tensor and the stress tensor. Each dumbbell induces a force-dipole flow in the surrounding solvent-the stress tensor is the ensemble average of this dipole. In the presence of a wall, the image of this force dipole induces a fluid velocity $\mathcal{M}: \tau^{p} / n$ at the position of the dumbbell; migration arises from the convection of the dumbbell due to this flow. ${ }^{54}$ Note that the term $\mathcal{M}: \tau^{p}$ is generic for the flux of any flexible suspended particle or molecule in a wall-bounded flow-in particular, its validity is not restricted to the dumbbell model. This term is missing in previous theories of polymer migration. The term containing the divergence of $\left\langle\mathbf{D}_{K, b}\right\rangle$ can also lead to migration, but only in a flow where the conformation distribution is spatially nonuniform (as in a pressure-driven flow) and only if the diffusivity of the molecule depends on conformation. As mentioned in the Introduction, several previous studies on the shear-induced migration in polymers focused on this term but neglected the hydrodynamic effect of the walls. ${ }^{14,46,63,64}$ In a pressure-driven flow, this term leads to a weak driving force toward the wall, but except at the centerline of the channel where the hydrodynamic migration term vanishes by symmetry, our previous simulations show that this effect is small. ${ }^{12}$ In nonhomogeneous flow, the term containing $\boldsymbol{\nabla \nabla \mathbf { v }}$ predicts the lag of a macromolecule behind the solvent along the streamline ${ }^{33}$ but no cross-streamline migration, and unless the nonhomogeneity is so large that it cannot be ignored even on the length scale of the polymer molecule, this term 
is small. Finally, the contribution to polymer flux proportional to $\boldsymbol{\nabla} \cdot \boldsymbol{\tau}^{p}$ predicted by several models ${ }^{26-32,34-37}$ does not arise in the single-molecule limit analyzed here.

\section{STEADY-STATE DEPLETION LAYER NEAR A SINGLE WALL}

Now consider an initially homogeneous infinitely dilute polymer solution under uniform shear flow in the $x$ direction with constant shear rate $\dot{\gamma}$ above an infinite plane wall at $y$ $=0$. Due to symmetry, no concentration variations will arise in the $x$ and $z$ directions; for the $y$ direction, using Eqs. (14) and (51), we have

$$
\frac{\partial n}{\partial t}=-\frac{\partial j_{c, y}}{\partial y}=-\frac{\partial}{\partial y}\left[\frac{K(y)}{y^{2}} n-D \frac{\partial n}{\partial y}\right],
$$

where

$$
\begin{aligned}
& K=\frac{3}{64 \pi \eta n}\left[\tilde{\mathcal{M}}: \tau^{p}\right]_{y}=\frac{3}{64 \pi \eta n}\left(N_{1}-N_{2}\right), \\
& D=\frac{k_{B} T}{12 \pi \eta a} .
\end{aligned}
$$

Here $N_{1}$ and $N_{2}$ are the first and second normal stress differences, defined by

$$
N_{1}=\tau_{x x}^{p}-\tau_{y y}^{p}, \quad N_{2}=\tau_{y y}^{p}-\tau_{z z}^{p} .
$$

In addition, for simplicity and because it is a good approximation for highly stretched dumbbells, we have replaced the conformation-dependent diffusivity by its free draining value $D$. Note that in Eq. (52) the migration velocity in the wallnormal direction is given by

$$
v_{\text {mig }}=\frac{K}{y^{2}}=\frac{3}{64 \pi \eta n y^{2}}\left(N_{1}-N_{2}\right) \text {. }
$$

This result is identical to that derived by Smart and Leighton for a suspended droplet. ${ }^{54}$

If we make the further assumption that $K$ is independent of position, again a good assumption for dilute solution in uniform shear, then Eq. (52) can be solved for the steadystate concentration profile,

$$
n=n_{b} \exp \left(-\frac{L_{d}}{y}\right)
$$

where $n_{b}$ is the bulk concentration and $L_{d}$ is the depletion layer thickness, defined by

$$
L_{d}=\frac{K}{D}=\frac{9}{16} \frac{N_{1}-N_{2}}{n k_{B} T} a .
$$

This quantity characterizes the length scale of the steadystate depletion layer in a semi-infinite domain. Note that for a long flexible molecule, $\left(N_{1}-N_{2}\right) / n k_{B} T$ can be much greater than unity. Since for a dumbbell model, the hydrodynamic bead radius $a$ is proportional to the molecular size, this result shows that depletion layers much thicker than the molecular size should be expected to arise in flows of dilute polymer solutions.
Equation (58) for the depletion layer thickness applies to any force law chosen for bead-spring model, since the spring force has been automatically built into the polymer contribution to the stress tensor $\tau^{p}$. Ideally, the evolution equation of the stress tensor would arise from the theory presented in Sec. IV, but the presence of the hydrodynamic interactions precludes the development of a closed-form equation. ${ }^{62}$ Therefore, to proceed with the analysis, we will use the FENE-P (finitely extensible nonlinear elastic-Peterlin) dumbbell model, which is simple, theoretically well understood, and widely used in simulations. ${ }^{65}$ The FENE-P spring force is given as follows:

$$
\mathbf{F}^{s}=\frac{H \mathbf{q}}{1-\left\langle q / q_{0}\right\rangle^{2}},
$$

where $q=|\mathbf{q}|, H$ is the spring constant, and $q_{0}$ is the maximum extended length of the dumbbell. The stress relaxation time is $\lambda_{H}=\zeta / 4 H$, where $\zeta=6 \pi \eta a$ is the bead friction coefficient. For $H q_{0}^{2} / k_{B} T \gg 1$, the radius of gyration is given by $R_{g}=\sqrt{k_{B} T / 2 H}$.

Introducing the length unit $\sqrt{k_{B} T / H}$ and time unit $\lambda_{H}$ $=\zeta / 4 H$, dimensionless quantities can be defined as

$$
\begin{aligned}
& \hat{\mathbf{q}}=\mathbf{q} / \sqrt{k_{B} T / H}, \quad \hat{t}=t / \lambda_{H}, \\
& \hat{\mathbf{v}}=\mathbf{v} \lambda_{H} / \sqrt{\left.k_{B} T / H\right)}, \quad b=H q_{0}^{2} / k_{B} T .
\end{aligned}
$$

For the FENE-P model, the dimensionless stress tensor then is

$$
\frac{\tau^{p}}{n k_{B} T}=\frac{\langle\hat{\mathbf{q}} \hat{\mathbf{q}}\rangle}{1-\left\langle\hat{\mathbf{q}}^{2}\right\rangle / b}-\mathbf{I} .
$$

The evolution equation for the structure tensor $\boldsymbol{\alpha}(\hat{t})=\langle\hat{\mathbf{q}} \hat{\mathbf{q}}\rangle$ is 65

$$
\frac{d \boldsymbol{\alpha}}{d \hat{t}}=\boldsymbol{\nabla} \hat{\mathbf{v}}^{T} \cdot \boldsymbol{\alpha}+\boldsymbol{\alpha} \cdot \nabla \hat{\mathbf{v}}-\frac{\boldsymbol{\alpha}}{1-\operatorname{tr}(\boldsymbol{\alpha}) / b}+\mathbf{I} .
$$

In addition to the Peterlin closure for the spring law, there are two approximations involved in using this equation for the stress. The first is the neglect of hydrodynamic interactions, either between the beads or between the dumbbell and the wall. The second is the neglect of transport of conformation due to diffusion and migration. The first approximation is necessary because it is impossible to get a closedform evolution equation for the structure tensor if full hydrodynamic interactions are included. The effect of this approximation is primarily to ignore a weak dependence of relaxation time with distance from the wall. We would like to point out that this approximation is invoked only when evaluating the numerical value of the depletion layer thickness where the stress value is needed. So the main physics (e.g., the migration mechanism, the expression for the centerof-mass flux, and the expression for depletion layer thickness) is free from this approximation. The effect of the second approximation will be negligible unless the velocity gradient varies on the scale of the molecular size, as migration and diffusion occur on a time scale much larger than the relaxation time. 


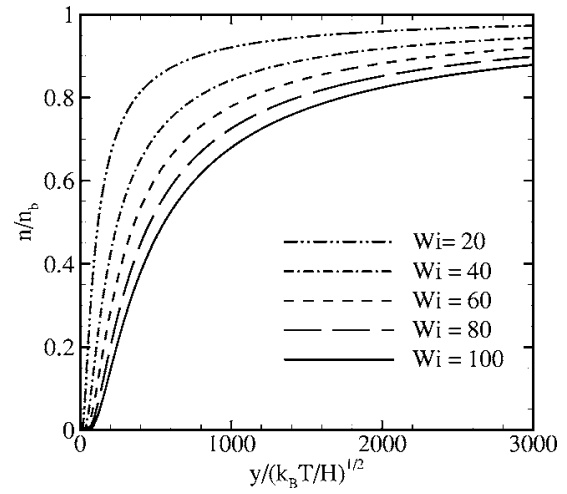

FIG. 3. Steady-state concentration profiles scaled by the bulk value in uniform shear flow above a single wall at different Weissenberg numbers. The concentration profiles are calculated using a FENE-P dumbbell model with finite extensibility parameter $b=600$ and hydrodynamic interaction parameter $h^{*}=0.25$.

Having specified the polymer model, we now return to the expression for the depletion layer thickness, Eq. (58). By introducing the hydrodynamic interaction parameter $h^{* 62}$

$$
h^{*}=\frac{\zeta}{\eta_{s}} \sqrt{\frac{H}{36 \pi^{3} k_{B} T}},
$$

the depletion layer thickness can be expressed as

$$
L_{d}=\frac{9 \sqrt{\pi}}{128} \frac{N_{1}-N_{2}}{n k_{B} T} h^{*} R_{g} .
$$

Now we define the Weissenberg number $\mathrm{Wi}=\lambda \dot{\gamma}$. The polymer contribution to the stress at different Weissenberg numbers can be calculated using FENE-P model Eq. (62). Figure 3 shows the steady-state concentration (probability) profile for different Weissenberg numbers when $b=600$ and $h^{*}$ $=0.25$ (these parameters will be used throughout this paper). The vertical axis is the concentration scaled by the bulk concentration $n_{b}$ and the horizontal axis is the distance from the wall scaled by $\sqrt{k_{B} T / H}$. Note that the depletion layer extends a very large distance from the wall, much larger than the size of the polymer molecule.

Finally, for a FENE-P dumbbell in shear flow, $\left(N_{1}\right.$ $-N_{2}$ ) $/ n k_{B} T$ scales as $\mathrm{Wi}^{2 / 3}$ at high Wi (Ref. 65) (this scaling also holds for the FENE dumbbell model without the closure approximation). Therefore, the depletion layer thickness scales as

$$
L_{d} \sim \mathrm{Wi}^{2 / 3} R_{g} .
$$

Figure 4 shows $L_{d}$ vs Wi on a $\log$-log scale for $b=600$ and $h^{*}=0.25$. The two-thirds power law at high $\mathrm{Wi}$ is evident. The result that $L_{d} \gg \sqrt{k_{B} T / H}$ for $\mathrm{Wi} \gg 1$ justifies our neglect of the wall exclusion in the model.

\section{TEMPORAL AND SPATIAL EVOLUTION OF THE DEPLETION LAYER IN A SEMI-INFINITE DOMAIN}

The above results show that at steady state, the hydrodynamic effect of a polymer with a wall leads to concentration variations on scales that can be orders of magnitude larger than the size of the polymer. We now turn to the tem-

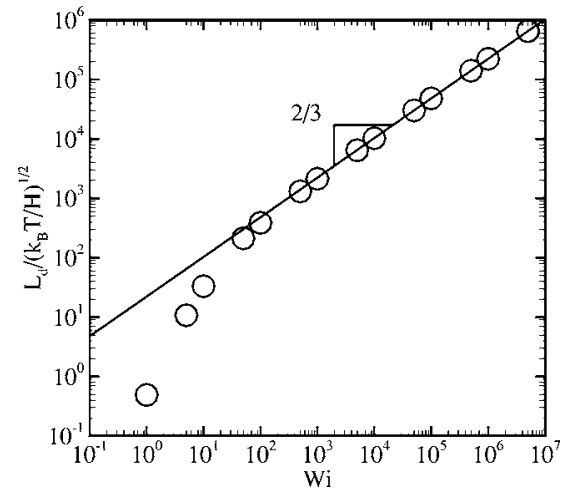

FIG. 4. Depletion layer thickness vs Weissenberg number in a uniform shear flow above a single wall for FENE-P dumbbell with finite extensibility parameter $b=600$ and hydrodynamic interaction parameter $h^{*}=0.25$. The straight line is the high Weissenberg number asymptote, $L_{d} / R_{g} \sim \mathrm{Wi}^{2 / 3}$.

poral and spatial developments of the depletion layer, beginning with the transient evolution of the concentration field in fluid above an infinite plane wall. At time $t=0$, the fluid begins to undergo uniform shear with shear rate $\dot{\gamma}$. The transient process is governed (under the same approximations as used above) by

$$
\frac{\partial n}{\partial t}=-\frac{\partial j_{c, y}}{\partial y}=-\frac{\partial}{\partial y}\left(\frac{K}{y^{2}} n\right)+D \frac{\partial^{2} n}{\partial y^{2}},
$$

initial condition: $n(y, 0)=n_{b}$,

$$
\text { boundary conditions: }\left\{\begin{array}{l}
j_{c, y}(0, t)=0, \\
n \rightarrow n_{b} \text { as } y \rightarrow \infty
\end{array} .\right.
$$

The time evolution of the concentration profile has been obtained by numerically solving this equation coupled with the stress evolution equation [Eq. (62)] using the FENE-P model, which determines $K(t)$. Figure 5 shows the concentration profile at different times for $\mathrm{Wi}=10, b=600$, and $h^{*}$ $=0.25$. This figure shows clearly that immediately after inception of the shear flow, a peak appears in concentration. This is simple to understand in terms of the dependence of migration rate on the distance from the wall. The migration

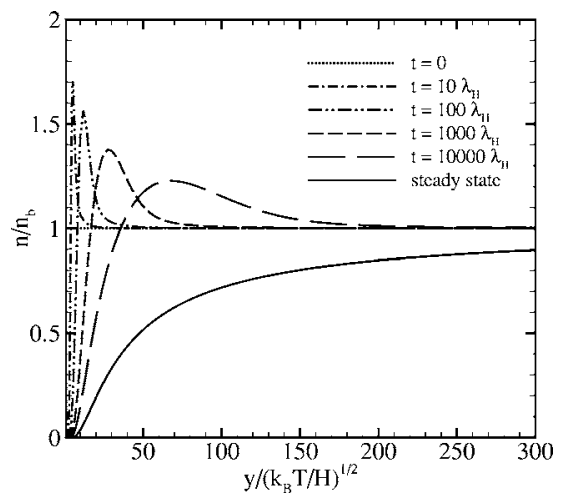

FIG. 5. Temporal development of the concentration profile in uniform shear flow above a single wall at $\mathrm{Wi}=10$. A FENE-P dumbbell model with finite extensibility parameter $b=600$ and hydrodynamic interaction parameter $h^{*}$ $=0.25$ is used 
rate [Eq. (56)] is larger near the wall than far from it. So the polymer molecules will "pile up." This effect is so dominant at short times that diffusion cannot smooth out the spike. At long times, however, as shown in the figure, the spike is smoothed out. The calculation of Hudson ${ }^{66}$ on the wall migration of fluid droplets in emulsions illustrated similar results.

The time scale involved in this process is remarkably large. Even after $10^{4}$ relaxation times, the steady state is still not reached. A simple estimate of the time required to reach steady state is given by the time $t_{\text {mig }}$ it takes for a molecule to migrate from $y=0$ to $y=L_{d}$,

$$
t_{\text {mig }} \sim \int_{0}^{L_{d}} \frac{d y}{v_{\text {mig }}} \sim \frac{L_{d}^{2}}{D} .
$$

By this estimate, the migration time scale is on the same order as that of the diffusion time over distance $L_{d}$. For the computation shown in Fig. 5, $L_{d}^{2} / D \approx 7 \times 10^{3}$; the results show that the simple estimate dramatically underpredicts the actual time required to approach steady state. This discrepancy arises because, as pointed out above, the depletion region, though characterized by $L_{d}$, is extremely broad-at $y$ $=L_{d}$, the steady-state concentration is only about $37 \%$ of $n_{b}$, the bulk concentration.

Further insight into the transient development of the depletion layer can be gained by considering the behavior at times much shorter than the diffusion time over the distance $L_{d}$ but long compared to $\lambda_{H}$, so $K$ in Eq. (66) can be treated as time independent. Introducing a transient depletion layer thickness $\delta_{y}(t)$, an order-of-magnitude analysis of Eq. (66) shows that at these short times the dominant balance is between the time-derivative term and the migration term, and that the depletion layer thickness scales as follows:

$$
\delta_{y}(t) \sim(K t)^{1 / 3} .
$$

Neglecting diffusion in Eq. (66) and defining the variable,

$$
\omega=\frac{y}{(3 K t)^{1 / 3}},
$$

a similarity solution can be found,

$$
\frac{n(\omega)}{n_{b}}= \begin{cases}0, & \text { if } 0 \leqslant \omega \leqslant 1 . \\ \frac{\omega^{2}}{\left(\omega^{3}-1\right)^{2 / 3}}, & \text { if } \omega>1 .\end{cases}
$$

This solution is shown in Fig. 6. Interestingly, it has an integrable singularity and discontinuity at $\omega=1$. The singularity arises because diffusion has been neglected completely, on the assumption that it is not important over the length scale $\delta_{y}(t)$. Moreover, in the absence of the diffusion all molecules escape from inside the depletion layer $(0 \leqslant \omega \leqslant 1)$, giving rise to the discontinuity at the frontier of the depletion layer $(\omega=1)$. Very near the singular point diffusion will become important at leading order, smearing out the singularity. Using the similarity solution to solve for $\omega$ values at which the migration contribution to the flux is comparable to the diffusion contribution, we found that the width of this region is proportional to $\delta_{y}(t) / L_{d}$. To illustrate this better, we calculate the full numerical solution to Eq. (66) by using a FENE-P

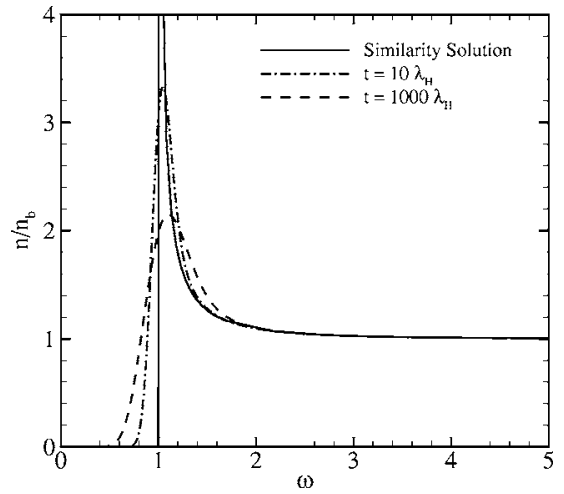

FIG. 6. Similarity solution for time evolution of the concentration profile in uniform shear flow above a single wall. The full numerical solutions including diffusion for $\mathrm{Wi}=100$ at two different times, $t=10 \lambda_{H}$ and $t=1000 \lambda_{H}$, are also plotted for comparison. A FENE-P dumbbell with finite extensibility $b=600$ and hydrodynamic interaction parameter $h^{*}=0.25$ is used when solving for the numerical solutions.

dumbbell model with finite extensibility $b=600$ and hydrodynamic interaction parameter $h^{*}=0.25$ at $\mathrm{Wi}=100$. The full numerical solutions at $t=10 \lambda_{H}$ and $1000 \lambda_{H}$ are plotted in Fig. 6 against the similarity solution. We see that the "pile up" phenomenon that occurs in the full numerical solution appears in idealized form in the similarity solution, showing its origin in the balance between migration and accumulation of the polymer, as described qualitatively above. Considering the large time difference (two orders of magnitude) between the two numerical solutions, the similarity solution captures the transient evolution of the depletion layer remarkably well. Finally, we note that the time scale for the development of the steady-state profile can be estimated from the scaling analysis by determining the time at which $\delta_{y}(t)=L_{d}$. This estimate recovers our earlier prediction that $t_{\mathrm{mig}} \sim L_{d}^{2} / D$.

Another important process is the spatial development of the concentration field near the entrance to a channel; we will address this situation here by considering the migration ana$\log$ of the Graetz-Leveque problem. ${ }^{67}$ At low Reynolds number, the velocity field near the entrance to the channel becomes fully developed over a length scale comparable to the height of the channel. Considering the region sufficiently near the channel entrance that the depletion layer is thin compared to the channel height $B$, we can treat the domain as semi-infinite in the $y$ direction and treat the velocity field as a simple shear flow. As above, flow is in the $x$ direction, and the wall is at $y=0$. The conservation equation becomes

$$
\dot{\gamma} y \frac{\partial n}{\partial x}=-\frac{\partial}{\partial y}\left(\frac{K}{y^{2}} n\right)+D\left(\frac{\partial^{2} n}{\partial x^{2}}+\frac{\partial^{2} n}{\partial y^{2}}\right) .
$$

Introducing a spatially varying depletion layer thickness $\delta_{y}(x)$, an order-of-magnitude analysis shows that very near the wall, the $y$-migration term and $x$-convection terms balance, and the scaling of the depletion layer thickness is given by 


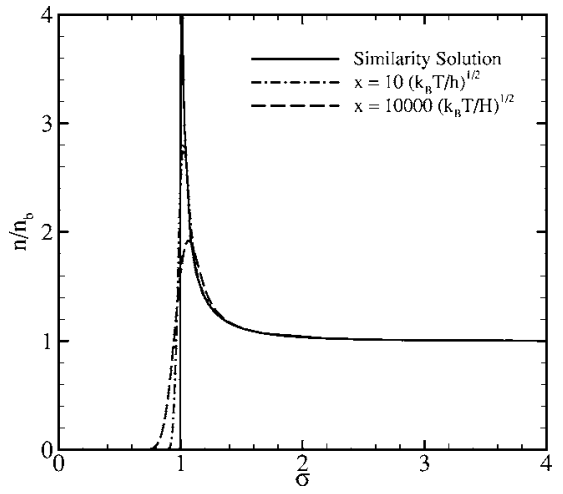

FIG. 7. Similarity solution for spatial development of the concentration profile in uniform shear flow above a single wall. The full numerical solutions including the diffusion for $\mathrm{Wi}=100$ at two different downstream positions, $x=10\left(k_{B} / H\right)^{1 / 2}$ and $x=10000\left(k_{B} / H\right)^{1 / 2}$, are also shown for comparison. A FENE-P dumbbell with finite extensibility $b=600$ and hydrodynamic interaction parameter $h^{*}=0.25$ is used when solving for the numerical solutions.

$$
\delta_{y}(x) \sim\left(\frac{K x}{\dot{\gamma}}\right)^{1 / 4} .
$$

Based on this scaling, we neglect the diffusion terms in Eq. (71) and seek a similarity solution $n(\sigma)$, where

$$
\sigma=\frac{y}{(4 K x / \dot{\gamma})^{1 / 4}}
$$

The solution is

$$
\frac{n(\sigma)}{n_{b}}= \begin{cases}0, & \text { if } 0 \leqslant \sigma \leqslant 1 . \\ \frac{\sigma^{2}}{\sqrt{\left|\sigma^{4}-1\right|}}, & \text { if } \sigma>1 .\end{cases}
$$

This solution is plotted in Fig. 7; for a channel with height $B$ it will be valid in the case $\delta_{h} \ll L_{d} \ll B$. Again, there is a weak singularity in this solution (which will be regularized by diffusion), showing that a pileup similar to that found in the transient development appears here too. So we expect that near the entrance to a channel the concentration distribution of polymer chains will display a peak near each wall. The assumption of negligible diffusion breaks down in a region around the singularity point $\sigma=1$ with width proportional to $\delta_{y}(x) / L_{d}$. The numerical solution without neglecting the $y$-diffusion term is solved by using a FENE-P dumbbell model with finite extensibility $b=600$ and hydrodynamic interaction parameter $h^{*}=0.25$ at $\mathrm{Wi}=100$, and the result is shown in Fig. 7 for downstream positions $x=10\left(k_{B} T / H\right)^{1 / 2}$ and $x=10000\left(k_{B} T / H\right)^{1 / 2}$. It is clear from the figure that the similarity solution captures the spatial development of the concentration field very well over a large length scale.

With the knowledge of the depletion layer thickness $L_{d}$ $=K / D$ in the fully developed region (i.e., where convection is negligible and diffusion and migration balance), a scaling estimate of the entrance length $L_{x}$ for the depletion layer can be obtained by setting the boundary layer thickness $\delta_{y}$ equal to $L_{d}$,

$$
\delta_{y}=\left(\frac{K L_{x}}{\dot{\gamma}}\right)^{1 / 4}=\frac{K}{D} .
$$

Therefore the entrance length is given by

$$
L_{x}=\frac{K^{3} \dot{\gamma}}{D^{4}}=L_{d}^{3} \frac{\dot{\gamma}}{D} .
$$

Combining with Eq. (65) and using the scaling relation $\lambda_{H}$ $\sim R_{g}^{2} / D$, we can rewrite $L_{x}$ in terms of $\mathrm{Wi}$ and $R_{g}$ for FENE dumbbells at high Wi as follows:

$$
L_{x} \sim \mathrm{Wi}^{3} R_{g} .
$$

This result shows that the entrance length is very sensitive to the Weissenberg number-a large entrance length should be expected at high Weissenberg number.

Finally, we address the issue of what residence time the fluid should have in the channel before the depletion layer can be considered to be fully developed. For the case of $L_{d}$ $\ll B$, this time can be estimated as the travel time from $x$ $=0$ to $x=L_{x}$ for a fluid element at a distance of $L_{d}$ from the wall,

$$
t_{\text {travel }} \sim \frac{L_{x}}{\dot{\gamma} L_{d}} \sim \frac{L_{d}^{2}}{D} .
$$

So roughly speaking, the residence time required for the establishment of the fully developed concentration profile is the diffusion time over the distance $L_{d}$. Based on the transient results presented above, however, we expect this estimate to underpredict the actual time required, because of the broad structure of the steady-state depletion layer. The experimentally obtained concentration profiles of Fang, $\mathrm{Hu}$, and Larson ${ }^{20}$ for DNA in a microchannel show a weak maximum, suggesting that they are not fully developed.

\section{PLANE COUETTE FLOW AND PLANE POISEUILLE FLOW}

The above analysis of shear-induced depletion in a semiinfinite domain reveals the basic mechanism of molecular migration and the time and length scales involved. In this section, we extend our discussion to a slit geometry and consider plane Couette flow and plane Poiseuille flow, which are very common flow types in experiments.

Consider the gap between two parallel plates separated by a distance $B$ and filled with polymer solution. As a first approximation, we can calculate the migration effects due to each wall in a semi-infinite domain with the other wall ignored and then superimpose the results. In this "single-reflection" 66 approximation, the total mass flux in the wall normal direction will be

$$
j_{c, y}=\frac{K(y)}{y^{2}} n-\frac{K(B-y)}{(B-y)^{2}} n-D \frac{\partial n}{\partial y} .
$$

The dependence of $K$ on position arises indirectly as a result of the position dependence of the shear rate. In plane Couette flow, where $K$ is position independent, the steady-state concentration profile under this approximation is 


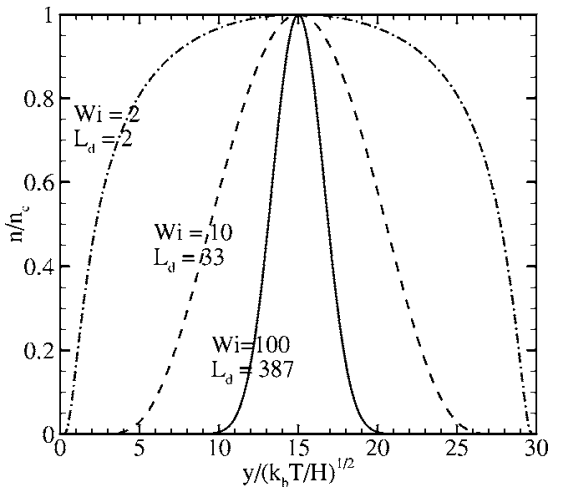

FIG. 8. Steady-state concentration profiles at $\mathrm{Wi}=2,10$, and 100 in plane Couette flow in a slit with width $B=30 \sqrt{k_{B} T / H}$. Length is scaled by $\sqrt{k_{B} T / H}$ and concentration by its value at the centerline of the slit, $n_{c}$. Migration effects due to the two walls of the slit are superimposed by taking the single-reflection approximation.

$$
\frac{n}{n_{c}}=\exp \left[-L_{d}\left(\frac{1}{y}+\frac{1}{B-y}-\frac{4}{B}\right)\right]
$$

where $L_{d}=K / D$ is the depletion layer thickness for an unbounded domain, $y$ is the distance from one wall, and $n_{c}$ is the concentration at the centerline of the slit. Figure 8 shows the solutions for $\mathrm{Wi}=2,10,100$, which correspond to $L_{d}$ $=2 \sqrt{k_{B} T / H}, 33 \sqrt{k_{B} T / H}$, and $387 \sqrt{k_{B} T / H}$. The parameters used are $b=600, h^{*}=0.25$, and $B=30 \sqrt{k_{B} T / H}$. As the Weissenberg number increases, the concentration profile becomes sharper and sharper, which indicates a stronger migration effect at higher Wi.

We now present results for plane Poiseuille flow. Here the velocity profile is parabolic,

$$
v_{x}(y)=U_{m}\left[1-\left(1-\frac{y}{B / 2}\right)^{2}\right],
$$

where $U_{m}$ is the velocity at the center of the slit. The steadystate concentration profiles in plane Poiseuille flow are shown in Fig. 9. Here Wi is defined based on the wall shear rate. In the middle of the slit, the concentration profile for plane Couette flow is steeper than that for plane Poiseuille

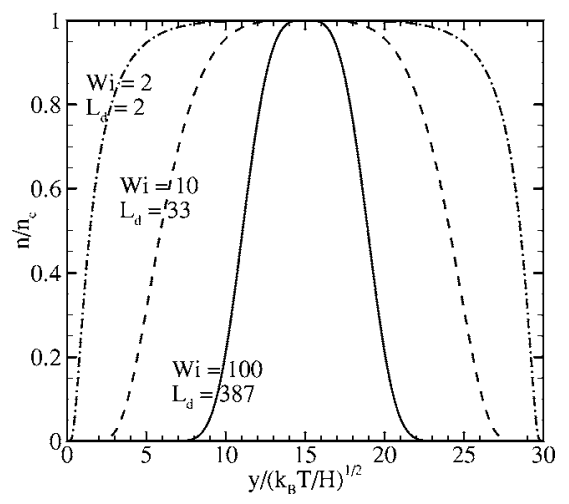

FIG. 9. Steady-state concentration profiles at $\mathrm{Wi}=2,10$, and 100 in plane Poiseuille flow in a slit with width $B=30 \sqrt{k_{B} T / H}$. Length is scaled by $\sqrt{k_{B} T / H}$ and concentration by its value at the centerline of the slit, $n_{c}$. Migration effects due to the two walls of the slit are superimposed by taking the single-reflection approximation.

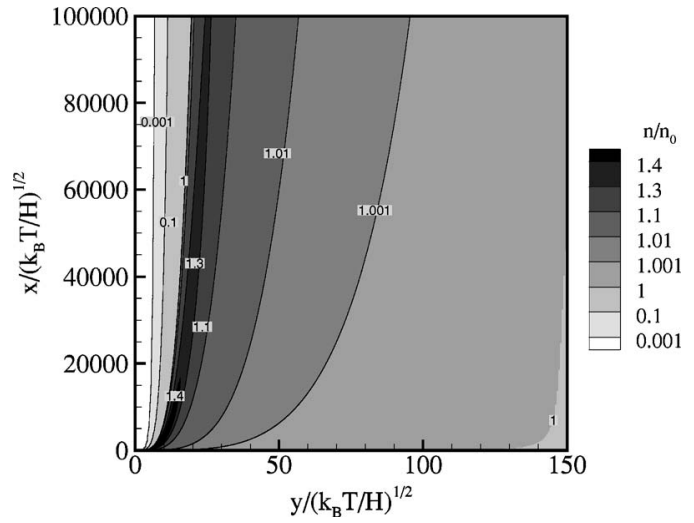

FIG. 10. Steady-state concentration field for plane Poiseuille flow in the entrance region of a slit with width $B=300 \sqrt{k_{B} T / H}$ at $\mathrm{Wi}=20$. Only half of the slit is shown. The concentration is scaled by its bulk value $n_{0}$ before entering the slit. Migration contributions due to two walls of the slit are superimposed by taking the single-reflection approximation.

flow. This is because the the shear rate in the middle region of the plane Couette flow is larger than that of plane Poiseuille flow.

A case of particular interest is the spatial development of the depletion layer downstream from the entrance to a slit, as we first discussed in Sec. VI. This situation is governed by the following equation:

$$
\begin{aligned}
v_{x}(y) \frac{\partial n}{\partial x}= & -\frac{\partial}{\partial y}\left[\frac{K(y)}{y^{2}} n\right]+\frac{\partial}{\partial y}\left[\frac{K(B-y)}{(B-y)^{2}} n\right] \\
& +D\left(\frac{\partial^{2} n}{\partial y^{2}}+\frac{\partial^{2} n}{\partial x^{2}}\right) .
\end{aligned}
$$

The numerical solution to this equation is shown in Fig. 10 for $B=300 \sqrt{k_{B} T / H}, b=600, h^{*}=0.25$, and $\mathrm{Wi}=20$. Concentration is scaled by its bulk value $n_{0}$ before entering the slit. It can be seen that the distance over which the concentration evolves into the fully developed profile is remarkably large, even for $x=10^{5} \sqrt{k_{B} T / H}$, the fully developed region is still not reached. This is consistent with the result from the similarity solution found in Sec. VI. Therefore, in order to measure the fully developed concentration in experiment, the residence time should be much larger than the diffusion time over the distance of the depletion layer thickness. The concentration field also shows clearly the pileup phenomenon, consistent with the similarity solution described above.

Finally, we note that there is one qualitative feature that is found in our detailed simulations of confined chains ${ }^{12}$ and predicted by Eq. (51), but not reproduced by the analysis presented in this section. This is the dip in the concentration profile at the center of the channel, which arises from the fourth term of Eq. (51), the migration toward regions of lower diffusivity that can arise in situations with a conformation-dependent diffusivity. This feature was lost due to our assumption of constant diffusivity. It would appear were we to use, for example, a model of diffusivity based on a deformation drag coefficient (see, e.g., Refs. 17, 43, 45, and 46). However, the effect is small in pressuredriven flow, ${ }^{12}$ and in uniform shear its only effect is to make $D$ dependent on Weissenberg number. 


\section{CONCLUSIONS}

In this paper, we developed a kinetic theory that describes migration phenomenon in flowing dilute polymer solutions near solid surfaces. The theory, which is based on a bead-spring dumbbell model of the polymer molecules, shows that the migration comes from two contributions: one that arises from the hydrodynamic interaction between the polymer molecule and the wall, and another that arises from intrachain hydrodynamic interactions. The first of these effects is generic for a flexible particle, droplet, or polymer molecule above a wall. Relaxation of the particle against the flow generates a force dipole, and if the particle is stretched and aligned parallel to the wall, the wall-normal flow induced by this force dipole convects the dumbbell away from the wall. The second effect is Brownian and drives the polymer molecules to regions of lower mobility. The latter effect is small in homogeneous shear flow, in which case the mobility of the polymer is virtually independent of position.

With this theory, we predict the steady-state concentration profile in uniform shear flow above an infinite plane wall. The profile, determined by the balance of migration and diffusion, has the form of a Boltzmann distribution and is characterized by a length scale $L_{d}$, the depletion layer thickness. The depletion layer thickness is proportional to the normal stress differences and the size of the polymer molecule. For FENE dumbbells at high Weissenberg number, $L_{d}$ $\sim \mathrm{Wi}^{2 / 3} R_{g}$, which can be much larger than the molecular size. In the transient development of the depletion layer, numerical simulations using the theory predict a spike on the concentration profile, which is corroborated by a similarity solution and can be explained by the dependence of the migration rate on the distance from the wall. The time scale for this transient process is shown to scale as the polymer diffusion time over the distance $L_{d}$. However, because of the extremely large breadth of the steady-state depletion layer, this estimate significantly underpredicts the actual time required to reach steady state. Using similar arguments, the entrance length $L_{x}$ for the concentration evolution in a channel is estimated (for FENE dumbbells) to scale with $\mathrm{Wi}^{3} R_{g}$. A spike in the spatially developing concentration profile also appears, as shown by numerical and similarity solutions. By taking the "single-reflection" approximation, the concentration profiles for plane Couette flow and plane Poiseuille flow are obtained.

The theory in its present form is only strictly valid for infinitely dilute solutions of dumbbells, though the dominant migration effect, the force-dipole interaction with the wall, is not restricted to the dumbbell model. At finite concentration, some hydrodynamic screening of the wall effect will occur, tending to weaken the migration effect, but on the other hand, because of the lower polymer concentration (and thus viscosity) near the wall, the shear rate will be higher there than in the bulk, tending to enhance migration. The balance of these effects will determine the concentration dependence of the depletion layer thickness and apparent slip velocity in dilute polymer solutions. The results presented here provide a starting point for addressing these issues.

\section{ACKNOWLEDGMENTS}

This work was supported by the National Science Foundation, Grant Nos. EES/BES/CTS-0085560 and DMR0425880 (Nanoscale Science and Engineering Center). The authors are grateful to J. J. de Pablo, D. C. Schwartz, E. Dimalanta, K. Jo, and Y.-L. Chen for many useful discussions.

${ }^{1}$ O. S. Andersen, "Sequencing and the single channel," Biophys. J. 77, 2899 (1999)

${ }^{2}$ C. F. Chou, R. H. Austin, O. Bakajin, J. O. Tegenfeldt, J. A. Castelino, S. S. Chan, E. C. Cox, H. Craighead, N. Darnton, T. Duke, J. Han, and S. Turner, "Sorting biomolecules with microdevices," Electrophoresis 21, 81 (2000).

${ }^{3}$ A. Lim, E. T. Dimalanta, K. D. Potamousis, G. Yen, J. Apodoca, C. Tao, J. Lin, R. Qi, J. Skiadas, A. Ramanathan, N. T. Perna, G. Plunkett III, V. Burland, B. Mau, J. Hackett, F. R. Blattner, T. S. Anantharaman, B. Mishra, and D. C. Schwartz, "Shotgun optical maps of the whole Escherichia coli O157: H7 genome," Genome Res. 11, 1584 (2001).

${ }^{4}$ N. Perna, G. Plunkett III, V. Burland, B. Mau, J. Glasner, D. Rose, G. Mayhew, P. Evans, J. Gregor, H. Kirkpatrick, G. Posfai, J. Hackett, S. Klink, A. Boutin, Y. Shao, L. Miller, E. Grotbeck, N. Davis, A. Lim, E. Dimalanta, K. Potamousis, J. Apodaca, T. Anantharaman, J. Lin, G. Yen, D. Schwartz, R. Welch, and F. Blattner, "Genome sequence of enterohaemorrhagic Escherichia coli O157: H7," Nature (London) 409, 529 (2001).

${ }^{5}$ M. Sauer, B. Angerer, W. Ankenbauer, Z. Földes-Papp, F. Göbel, K. T. Han, R. Rigler, A. Schultz, J. Wolfrum, and C. Zander, "Single molecule DNA sequencing in submicrometer channels: State of the art and future prospects," J. Biotechnol. 86, 181 (2001).

${ }^{6}$ M. Hinz, S. Gura, B. Nitzan, S. Margel, and H. Seliger, "Polymer support for exonucleolytic sequencing," J. Biotechnol. 86, 281 (2001).

${ }^{7}$ J. O. Tegenfeldt, C. Prinz, H. Cao, R. L. Huang, R. H. Austin, S. Y. Chou, E. C. Cox, and J. C. Sturm, "Micro- and nanofluidics for DNA analysis," Anal. Bioanal. Chem. 378, 1678 (2004).

${ }^{8}$ M. Chopra and R. G. Larson, "Brownian dynamics simulations of isolated polymer molecules in shear flow near adsorbing and nonadsorbing surfaces," J. Rheol. 46, 831 (2002).

${ }^{9}$ O. B. Bakajin, T. A. J. Duke, C. F. Chou, S. S. Chan, R. H. Austin, and E. C. Cox, "Electrohydrodynamic stretching of DNA in confined environments," Phys. Rev. Lett. 80, 2737 (1998).

${ }^{10}$ R. M. Jendrejack, D. C. Schwartz, M. D. Graham, and J. J. de Pablo, "Effect of confinement on DNA dynamics in microfluidic devices," J. Chem. Phys. 119, 1165 (2003).

${ }^{11}$ R. M. Jendrejack, E. T. Dimalanta, D. C. Schwartz, M. D. Graham, and J. J. de Pablo, "DNA dynamics in a microchannel," Phys. Rev. Lett. 91, 038102 (2003).

${ }^{12}$ R. M. Jendrejack, D. C. Schwartz, J. J. de Pablo, and M. D. Graham, "Shear-induced migration in flowing polymer solutions: Simulation of long-chain DNA in microchannels," J. Chem. Phys. 120, 2513 (2004).

${ }^{13}$ P. J. Shrewsbury, D. Liepmann, and S. J. Muller, "Concentration effects of a biopolymer in a microfluidic device," Biomed. Microdevices 4, 17 (2002).

${ }^{14}$ N. J. Woo, E. S. G. Shaqfeh, and B. Khomami, "Effect of confinement on dynamics and rheology of dilute DNA solutions. I. Entropic spring force under confinement and a numerical algorithm," J. Rheol. 48, 281 (2004).

${ }^{15}$ N. J. Woo, E. S. G. Shaqfeh, and B. Khomami, "The effect of confinement on dynamics and rheology of dilute deoxyribose nucleic acid solutions. II. Effective rheology and single chain dynamics," J. Rheol. 48, 299 (2004).

${ }^{16}$ Handbook of Micro/Nano Tribology, 2nd ed., edited by B. Bhushan (CRC, Boca Raton, FL, 1998).

${ }^{17}$ U. S. Agarwal, A. Dutta, and R. A. Mashelkar, "Migration of macromolecules under flow: The physical origin and engineering implications," Chem. Eng. Sci. 49, 1693 (1994)

${ }^{18}$ R. G. Horn, O. I. Vinogradova, M. E. Mackay, and N. Phan-Thien, "Hydrodynamic slippage inferred from thin-film drainage measurements in a solution of nonadsorbing polymer," J. Chem. Phys. 112, 6424 (2000).

${ }^{19}$ Y. Cohen and A. B. Metzner, "Apparent slip flow of polymer solutions," J. Rheol. 29, 67 (1985).

${ }^{20}$ L. Fang, H. Hu, and R. G. Larson, "DNA configurations and concentrations in shearing flow near a glass surface in a microchannel," J. Rheol. 49, 127 (2005). 
${ }^{21}$ F. H. Garner and A. H. Nissan, "Rheological properties of high viscosity solutions of long molecules," Nature (London) 158, 634 (1946).

${ }^{22} \mathrm{G}$. Marrucci, "The free energy constitutive equation for polymer solutions from the dumbbell model," Trans. Soc. Rheol. 16, 321 (1972).

${ }^{23}$ A. B. Metzner, in Improved Oil Recovery by Surfactant and Polymer Flooding, edited by D. O. Shah and R. S. Schechter (Academic, New York, 1977), p. 439.

${ }^{24}$ M. Tirrell and M. F. Malone, "Stress induced diffusion of macromolecules," J. Polym. Sci., Polym. Phys. Ed. 15, 1569 (1977).

${ }^{25}$ J. H. Aubert, S. Prager, and M. Tirrell, "Macromolecules in nonhomogeneous velocity gradient fields. II," J. Chem. Phys. 73, 4103 (1980).

${ }^{26}$ E. Helfand and G. H. Fredrickson, "Large fluctuations in polymer solutions under shear," Phys. Rev. Lett. 62, 2468 (1989).

${ }^{27}$ M. Doi, in Dynamics and Patterns in Complex Fluids: New Aspects of Physics and Chemistry of Interfaces, edited by A. Onuki and K. Kawasaki (Springer, Berlin, 1990), p. 100.

${ }^{28}$ A. Onuki, "Dynamic equations of polymers with deformations in semidilute regions," J. Phys. Soc. Jpn. 59, 3423 (1990).

${ }^{29}$ S. T. Milner, "Hydrodynamics of semidilute polymer solutions," Phys. Rev. Lett. 66, 1477 (1991).

${ }^{30}$ V. G. Mavrantzas and A. N. Beris, "Modeling of the rheology and flowinduced concentration changes in polymer solutions," Phys. Rev. Lett. 69, 273 (1992)

${ }^{31}$ S. T. Milner, "Dynamical theory of concentration fluctuations in polymer solutions under shear," Phys. Rev. E 48, 3674 (1993).

${ }^{32}$ W. B. Black and M. D. Graham, "Slip, concentration fluctuations, and flow instability in sheared polymer solutions," Macromolecules 24, 5731 (2001).

${ }^{33}$ J. H. Aubert and M. Tirrell, "Macromolecules in nonhomogeneous velocity gradient fields," J. Chem. Phys. 72, 2694 (1980).

${ }^{34}$ A. V. Bhave, R. C. Armstrong, and R. A. Brown, "Kinetic theory and rheology of dilute, nonhomogeneous polymer solutions," J. Chem. Phys. 95, 2988 (1991).

${ }^{35}$ H. C. Öttinger, "Incorporation of polymer diffusivity and migration into constitutive equations," Rheol. Acta 31, 14 (1992).

${ }^{36}$ A. N. Beris and V. G. Mavrantzas, "On the compatibility between various macroscopic formalisms for the concentration and flow of dilute polymer solutions," J. Rheol. 38, 1235 (1994).

${ }^{37}$ C. F. Curtiss and R. B. Bird, "Statistical mechanics of transport phenomena: Polymeric liquid mixtures," Adv. Polym. Sci. 125, 1 (1996).

${ }^{38}$ C. F. Curtiss and R. B. Bird, "Diffusion-stress relations in polymer mixtures," J. Chem. Phys. 111, 10362 (1999).

${ }^{39}$ J. H. Aubert and M. Tirrell, "Effective viscosity of dilute polymer solutions near confining boundaries," J. Chem. Phys. 77, 553 (1982).

${ }^{40}$ V. G. Mavrantzas and A. N. Beris, "Theoretical study of wall effects on the rheology of dilute polymer solutions," J. Rheol. 36, 175 (1992).

${ }^{41}$ V. G. Mavrantzas and A. N. Beris, "A hierarchical model for surface effects on chain conformation and rheology of poymer solutions. I. General formulation," J. Chem. Phys. 110, 616 (1999).

${ }^{42}$ V. G. Mavrantzas and A. N. Beris, "A hierarchical model for surface effects on chain conformation and rheology of poymer solutions. II. Application to a neutral surface," J. Chem. Phys. 110, 628 (1999).

${ }^{43}$ G. Sekhon, R. C. Armstrong, and M. S. Jhon, "The origin of polymer migration in a nonhomogeneous flow field," J. Polym. Sci., Polym. Phys. Ed. 20, 947 (1982).

${ }^{44} \mathrm{P}$. O. Brunn, "Hydrodynamically induced cross-stream migration of dissolved macromolecules (modelled as non-linearly elastic dumbbells)," Int. J. Multiphase Flow 9, 187 (1983).
${ }^{45}$ P. O. Brunn, "Polymer migration phenomena based on the general beadspring model for flexible polymers," J. Chem. Phys. 80, 5821 (1984).

${ }^{46} \mathrm{P}$. O. Brunn and S. Chi, "Macromolecules in non-homogeneous flow fields: A general study for dumbbell model macromolecules," Rheol. Acta 23, 163 (1984).

${ }^{47}$ M. S. Jhon and K. F. Freed, "Polymer migration in Newtonian fluids," J. Polym. Sci., Polym. Phys. Ed. 23, 955 (1985).

${ }^{48}$ X. Fan, N. Phan-Thien, N. T. Yong, X. Wu, and D. Xu, "Microchannel flow of a macromolecular suspension," Phys. Fluids 15, 11 (2003).

${ }^{49} \mathrm{P}$. Español and P. Warren, "Statistical mechanics of dissipative particle dynamics," Europhys. Lett. 30, 191 (1995).

${ }^{50}$ R. D. Groot and P. B. Warren, "Dissipative particle dynamics: Bridging the gap between atomic and mesoscopic simulation," J. Chem. Phys. 107, 4423 (1997).

${ }^{51}$ M. Ripoll, M. H. Ernst, and P. Español, "Large scale and mesoscopic hydrodynamics for dissipative particle dynamics," J. Chem. Phys. 115, 7271 (2001).

${ }^{52}$ L. G. Leal, "Particle motions in a viscous fluid," Annu. Rev. Fluid Mech. 12, 435 (1980).

${ }^{53}$ P. C. H. Chan and L. G. Leal, "The motion of a deformable drop in a second-order fluid,” J. Fluid Mech. 92, 131 (1979).

${ }^{54}$ J. R. Smart and D. T. Leighton, "Measurement of the drift of a droplet due to the presence of a plane," Phys. Fluids A 3, 21 (1991).

${ }^{55}$ R. M. Jendrejack, M. D. Graham, and J. J. de Pablo, "Hydrodynamic interactions in long chain polymers: Application of the Chebyshev polynomial approximation in stochastic simulations," J. Chem. Phys. 113, 2894 (2000).

${ }^{56}$ R. M. Jendrejack, J. J. de Pablo, and M. D. Graham, "Stochastic simulations of DNA in flow: Dynamics and the effects of hydrodynamic interactions," J. Chem. Phys. 116, 7752 (2002).

${ }^{57}$ F. Brochard and P. G. de Gennes, "Dynamics of confined polymer chains," J. Chem. Phys. 67, 52 (1977).

${ }^{58}$ Y.-L. Chen, M. D. Graham, J. J. de Pablo, G. C. Randall, M. Gupta, and P. S. Doyle, "Conformation and dynamics of single DNA in parallel-plate slit microchannels," Phys. Rev. E 70, 060901 (2004).

${ }^{59}$ Y.-L. Chen, M. D. Graham, J. J. de Pablo, K. Jo, and D. C. Schwartz, "DNA molecules in microfluidic oscillatory flow," Macromolecules 38, 6680 (2005).

${ }^{60}$ C. Pozrikidis, Introduction to Theoretical and Computational Fluid Dynamics (Oxford University Press, New York, 1997).

${ }^{61}$ J. R. Blake, "A note on the image system for a stokeslet in a no-slip boundary," Proc. Cambridge Philos. Soc. 70, 303 (1971).

${ }^{62}$ R. B. Bird, C. F. Curtiss, R. C. Armstrong, and O. Hassager, Dynamics of Polymeric Liquids, 2nd ed. (Wiley, New York, 1987), vol. 2.

${ }^{63}$ G. G. Fuller and L. G. Leal, "The effects of conformation-dependent friction and internal viscosity on the dynamics of the nonlinear dumbbell model for a dilute polymer solution," J. Non-Newtonian Fluid Mech. 8, 271 (1981)

${ }^{64}$ Y. H. Seo, O. O. Park, and M. S. Chun, "The behavior of velocity enhancement in microcapillary flows of flexible water-soluble polymers," J. Chem. Eng. Jpn. 29, 611 (1996).

${ }^{65} \mathrm{M}$. Herrchen and H. C. Öttinger, "A detailed comparison of various FENE dumbbell models," J. Non-Newtonian Fluid Mech. 68, 17 (1997).

${ }^{66}$ S. D. Hudson, "Wall migration and shear-induced diffusion of fluid droplets in emulsions," Phys. Fluids 15, 1106 (2003).

${ }^{67}$ L. G. Leal, Laminar Flow and Convective Transport Processes (Butterworth-Heinemann, Boston, 1992). 